\title{
TRI-BAND COMPACT ANTENNA ARRAY FOR MIMO USER MOBILE TERMINALS AT GSM 1800 AND WLAN BANDS
}

\begin{abstract}
Carlos Gómez-Calero, Luis González, and Ramón Martínez Department of Signals, Systems and Radiocommunications, Radiation Group, Universidad Politécnica de Madrid. 28040 Madrid, Spain; Corresponding author: carlosgc@gr.ssr.upm.es
\end{abstract}

Key words: planar inverted F-antenna; MIMO; WLAN; capacity

\begin{abstract}
Multiple-Input Multiple-Output (MIMO) systems have been widely studied in recent years as a solution to improve the radio link reliability. To introduce MIMO in mobile communications, it is necessary to design antennas with differential features to introduce multiple elements in the space reserved for the antenna, such as the type of element or array configuration. In this article, a $2 \times 2$ MIMO system has been considered according to the next-generation WLAN (802.11n) frequencies band.
\end{abstract}

\section{INTRODUCTION}

In recent years, the exploitation of space domain has been considered as a very interesting way to increase the capacity of wireless systems. New methods and schemes to make use of the channel spatial diversity have been considered, such as the use of multiple antennas in both sides of the communication link, popularly known as MIMO (multiple-input multiple-output) systems The achievable performance in a MIMO system depends mainly on three aspects, namely the radio channel propagation characteristics, the MIMO algorithms used in transmission and reception, and finally the antenna array configuration by means of its radiation and polarization characteristics

Regarding the antenna aspects, many compact designs have been studied to obtain different resonant frequencies for multiband antennas, as it is detailed in [3]. Meander lines, folded patches, and slots are introduced to achieve the desired bands, being the planar inverted-F antenna (PIFA) an interesting solution. Because the antenna for mobile applications has to be located in the user terminal, the dimensions of the radiating element creates certain constrains and the trade off between performances and miniaturization. On the other hand, new MIMO techniques need the use of several antennas in transmission and reception. As far as the array is concerned, different aspects such as coupling and radiation pattern have to be taken into account when MIMO techniques are used.

In this article, a $2 \times 2$ MIMO antenna system has been designed, implemented, and measured for the next-generation WLAN (802.11n) and GSM. Two PIFAs for GSM and WLAN bands $(1800 / 2400 / 5200 \mathrm{MHz})$ have been designed for a PDA terminal. This type of antenna has been chosen regarding the small space in user terminals. The results have been taken into account to obtain the MIMO channel capacity including electromagnetic parameters such as the radiation pattern and mutual coupling, depending on the employed configuration. Different configurations have been evaluated and the best one in performances has been implemented and introduced in a MIMO testbed to obtain channel measurements and compare it with a reference MIMO array.

\section{ANTENNA DESIGN}

Several design aspects must be taken into account when PIFAs are designed. On the one hand, the dielectric between the ground plane and the radiating patch affects on the frequency response, efficiency, and radiation pattern. On the other hand, for the sake of simplicity, a pin is introduced in spite of a wall to make the short-circuit. Regarding this, the dimensions of the overall antenna when the width of the short-circuit is close to zero, is given by

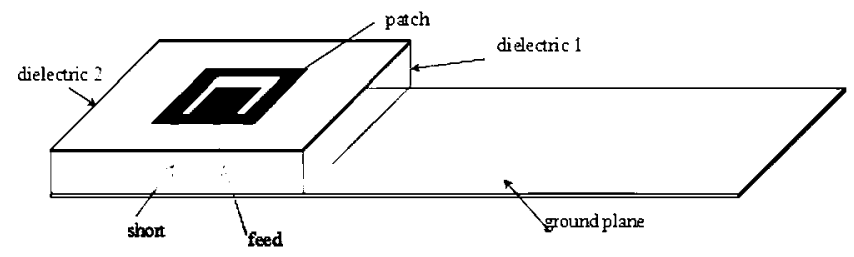

Figure 1 Antenna structure for the design in a user terminal 


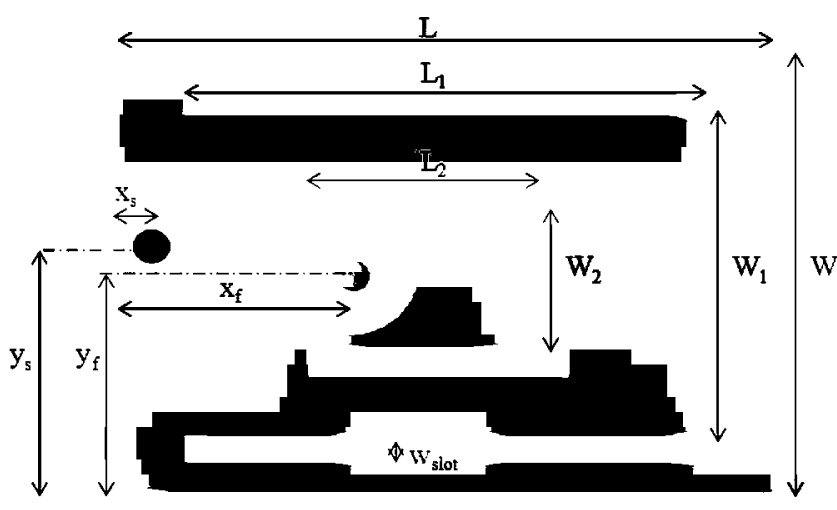

Figure 2 Dimensions of the radiating match with the two U-shaped slots, where $L=21, W=15, L_{1}=18, W_{1}=13, L_{2}=8.5, W_{2}=7, W_{\text {slot }}$ $=1, x_{\mathrm{f}}=7, y_{\mathrm{f}}=7, x_{\mathrm{s}}=1$, and $y_{\mathrm{s}}=8.5$ (dimensions in $\mathrm{mm}$ )

$$
L+W+H=\frac{\lambda}{4}
$$

where $L$ and $W$ are the length and width of the patch, respectively, and $H$ represents the height between the ground plane and radiating patch.

The antenna structure is shown in Figure 1, where the configuration of the layers is detailed. First, the ground plane is situated in the bottom of the antenna. Above, the dielectric 1 is made of Rohacell foam with a dielectric constant $\left(\varepsilon_{\mathrm{r}}\right)$ of 1.05 . The second dielectric is fiber glass $\left(0.9 \mathrm{~mm}\right.$ of thickness and $\left.\varepsilon_{\mathrm{r}}=4.1\right)$ and supports the printed patch. Finally, two vias go through the two dielectric layers from the ground plane to the patch: short and feed. The dimensions of the antenna are $315 \mathrm{~mm}^{2}$, suitable to be installed in a handheld mobile terminal.

Two U-shaped slots have been placed in order to achieve the three resonant desired frequencies: $1.8,2.45$, and $5.4 \mathrm{GHz}$ for GSM and the two WLAN bands, respectively. The external patch is rectangular whose dimensions are represented in Figure 2. On the other hand, the feed and the short are situated in $x_{\mathrm{f}}, y_{\mathrm{f}}, x_{\mathrm{s}}$, and $y_{\mathrm{s}}$, in order to enhance the reflection coefficient and a linear polarization in the center of the patch.

\section{MIMO CONFIGURATIONS}

Regarding a $2 \times 2$ MIMO system, several array configurations have been simulated in a device with a size typical for high-end mobile applications: a PDA of $70 \times 110 \mathrm{~mm}^{2}$. Figure 3 shows the three configurations simulated. The PIFAs are placed varying $x$ and $y$ axis and rotated, reserving space for the device battery
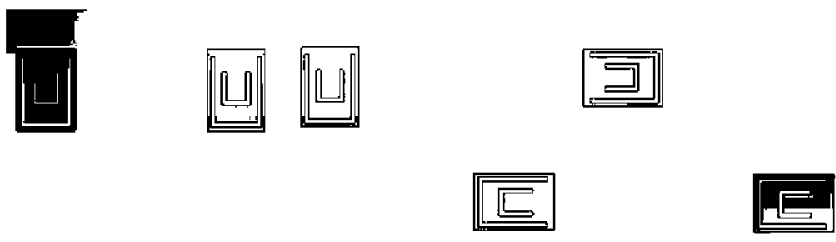

Figure 3 MIMO configurations for a PDA terminal: 1, 2, and 3 (from left to right). The bottom side is reserved for the battery of the terminal

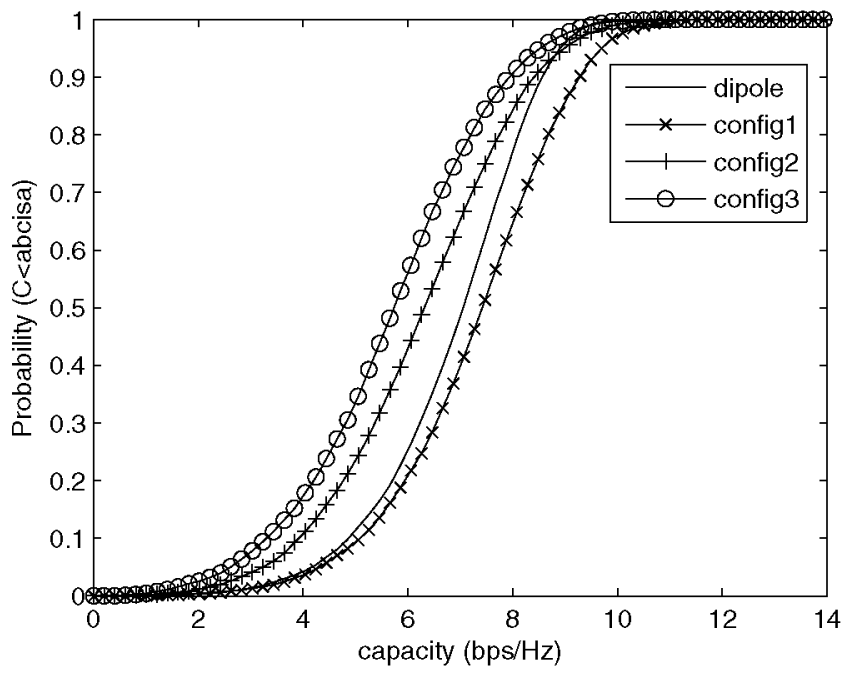

Figure 4 Cumulative Distribution Function of the capacity simulated for the three MIMO configurations compared to a dipole array

(bottom side of each figure). These changes in the position of each antenna generate different radiation patterns and mutual coupling between elements. It is worth notice here that all the antenna simulations have been carried out using CST Microwave Studio.

Once electromagnetic simulations have been performed, radiation patterns and mutual coupling characteristics are exported to a link level simulator to obtain the MIMO channel capacity. This simulator incorporates the $3 \mathrm{GPP} 2$ channel model implementation

The channel has been configured with a cell radius of $100 \mathrm{~m}$ and taking into account the polarizations with an $\mathrm{AoD}_{\text {max }}=180^{\circ}$ to simulate indoor environments. Once the wideband channel

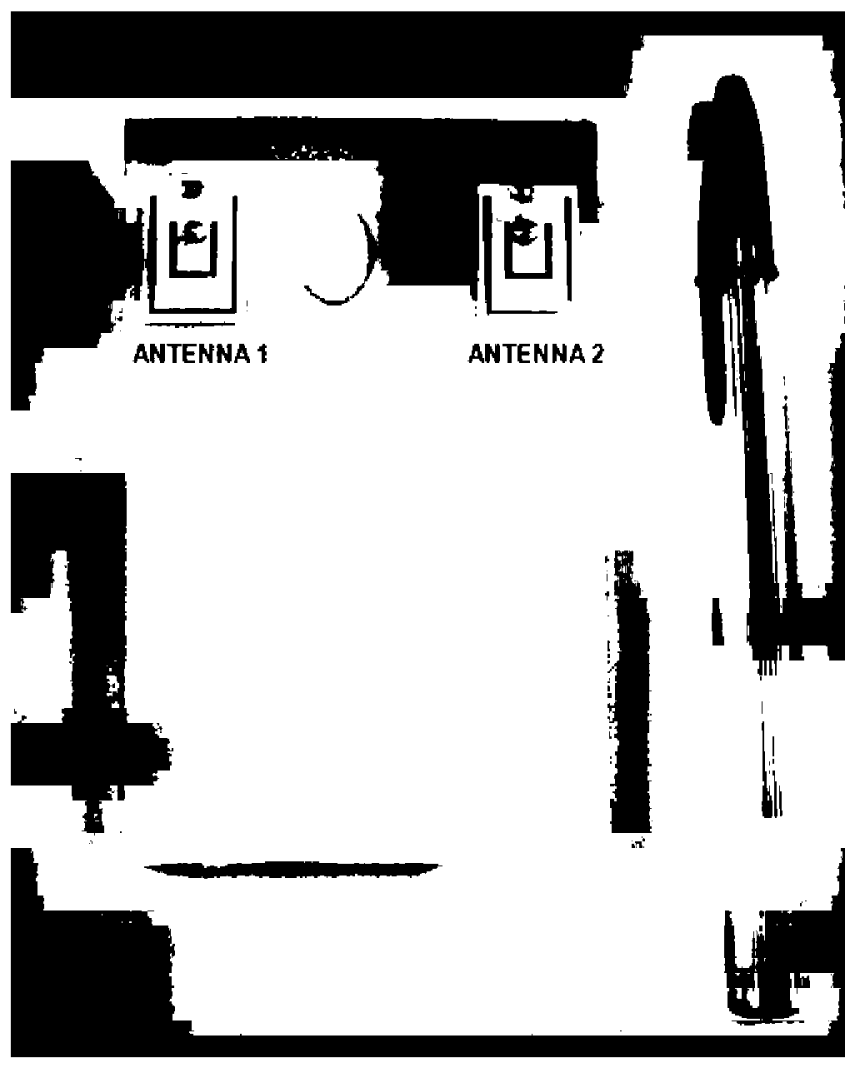

Figure 5 Final implementation of MIMO antenna array configuration 


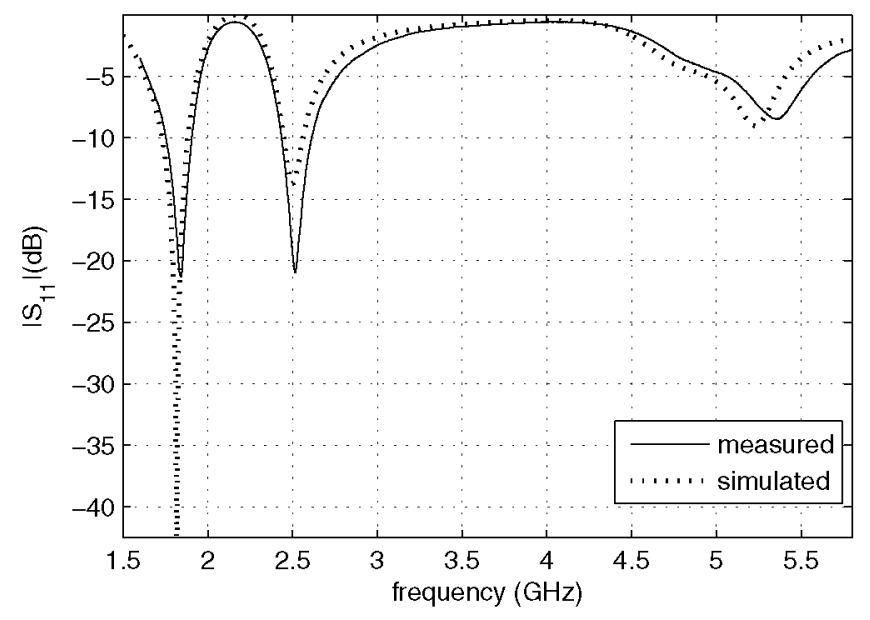

Figure 6 Simulated and measured reflection coefficients $\left(S_{11}\right)$ for the antenna 1

matrix $H(t, \tau)$ is obtained from the SCM, an equivalent narrowband channel matrix is computed for $f=2.45 \mathrm{GHz}$ given by

$$
H(t)=\sum_{n=1}^{L} H\left(t, \tau_{n}\right) e^{\left(-j \cdot 2 \pi \cdot f o \cdot \tau_{n}\right)}
$$

where $L$ is the number of paths, which in this case is six. The capacity is calculated by (3), where $\mathbf{I}_{\mathrm{MR}}$ is the eye matrix, $\rho$ represents the signal to noise ratio, $M_{\mathrm{T}}$ indicates the number of transmitter antennas, $M_{\mathrm{R}}$ the number of receiver antennas, $\mathbf{H}$ is the channel matrix including antenna radiation patterns, and $\mathbf{C}_{\mathbf{R}}$ and $\mathbf{C}_{\mathbf{T}}$ are the multi-element antenna (MEA) correlation matrices in the receiver and transmitter. It should be noted that this expression is only valid under a number of assumptions, which include perfect channel knowledge at the receiver and the same transmitted power regardless of the number of transmit antennas $\left(M_{\mathrm{T}}\right)$.

$$
C=\log _{2}\left|\mathbf{I}_{M_{\mathrm{R}}}+\frac{\rho}{M_{\mathrm{T}}} \mathbf{C}_{\mathbf{R}} \mathbf{H} \mathbf{H}^{I[b]} C_{T}\right| \quad \operatorname{bps} / \mathrm{Hz}
$$

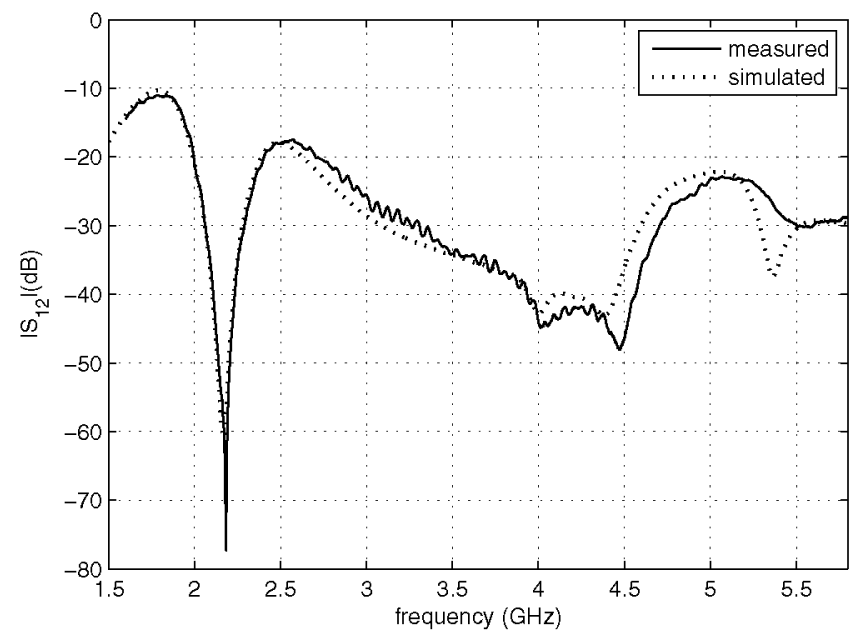

Figure 7 Simulated and measured coupling coefficients $\left(S_{12}\right)$ for the antenna 1

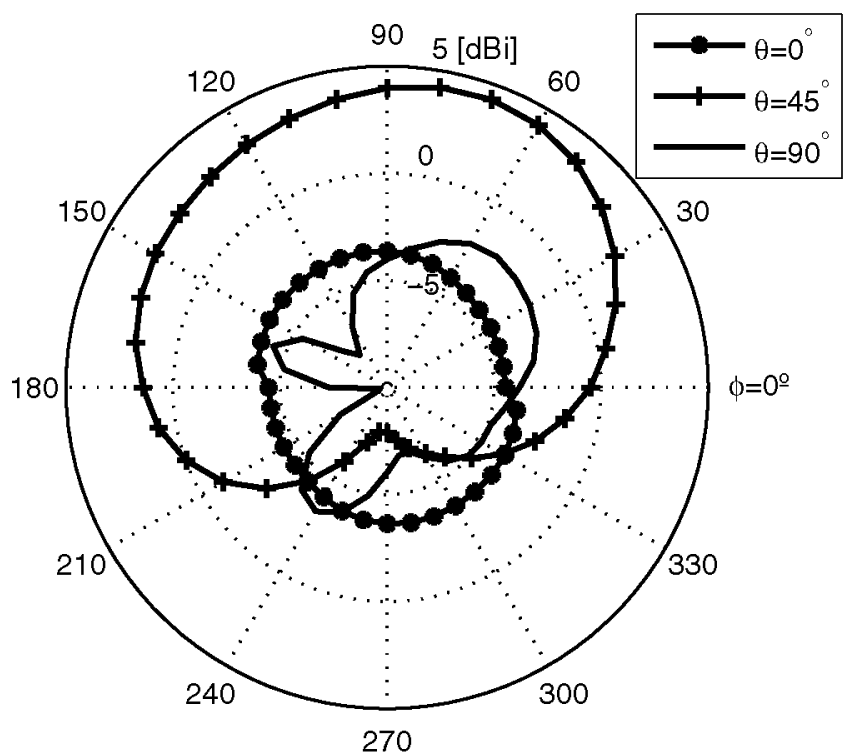

Figure 8 Radiation pattern measured for the antenna 1

$\mathbf{C}_{\mathbf{R}}$ is given by (4), where $\mathbf{S}$ represents $S$ parameters matrix obtained from simulated results in CST. In this case, $\mathbf{C}_{\mathbf{T}}=\mathbf{I}_{\mathrm{MT}}$ since the aim is to study the effects only in user terminal.

$$
\mathbf{C}_{\mathbf{R}}=\mathbf{I}_{M_{\mathrm{R}[b]}}-\mathrm{S}^{H[b]} S
$$

Figure 4 depicts the cumulative distribution function (CDF) of the capacity obtained for several configurations in the case of the tri-band antenna for an elevation angle of $\theta=45^{\circ}$. This is due to the position of the user terminal and the transmitter one in an indoor environment. As it is depicted, the configuration 1 offers higher capacity for the points of probability, even higher than the dipole case. The capacity depends on the correlation between antennas and the configuration 1 have lower correlation because of the mutual coupling and the antenna gain for $\left(\varphi, \theta=45^{\circ}\right)$.

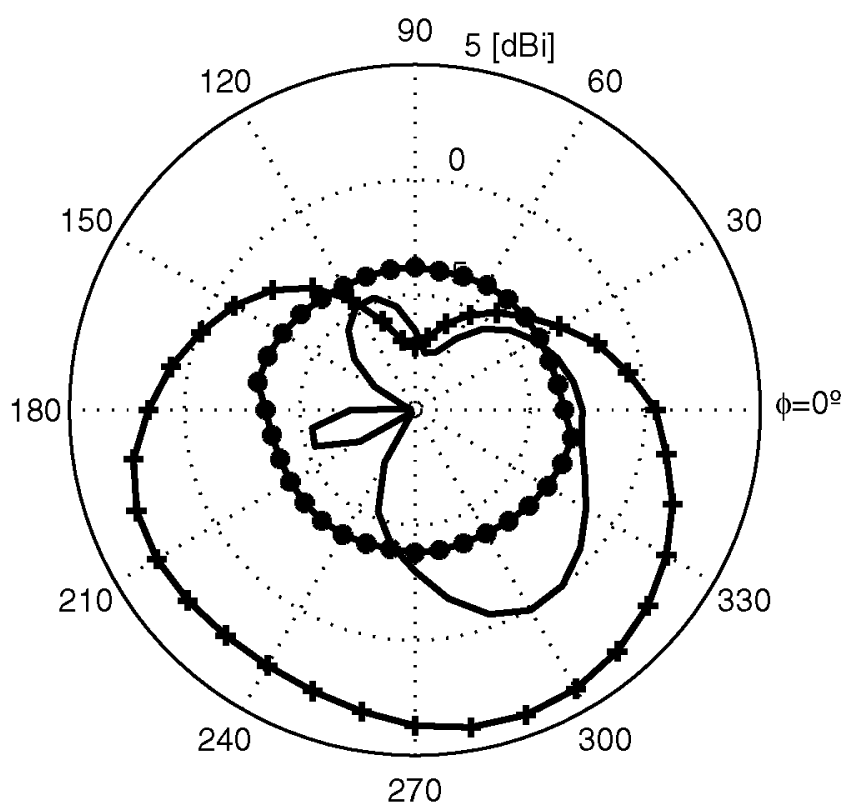

Figure 9 Radiation pattern measured for the antenna 2 


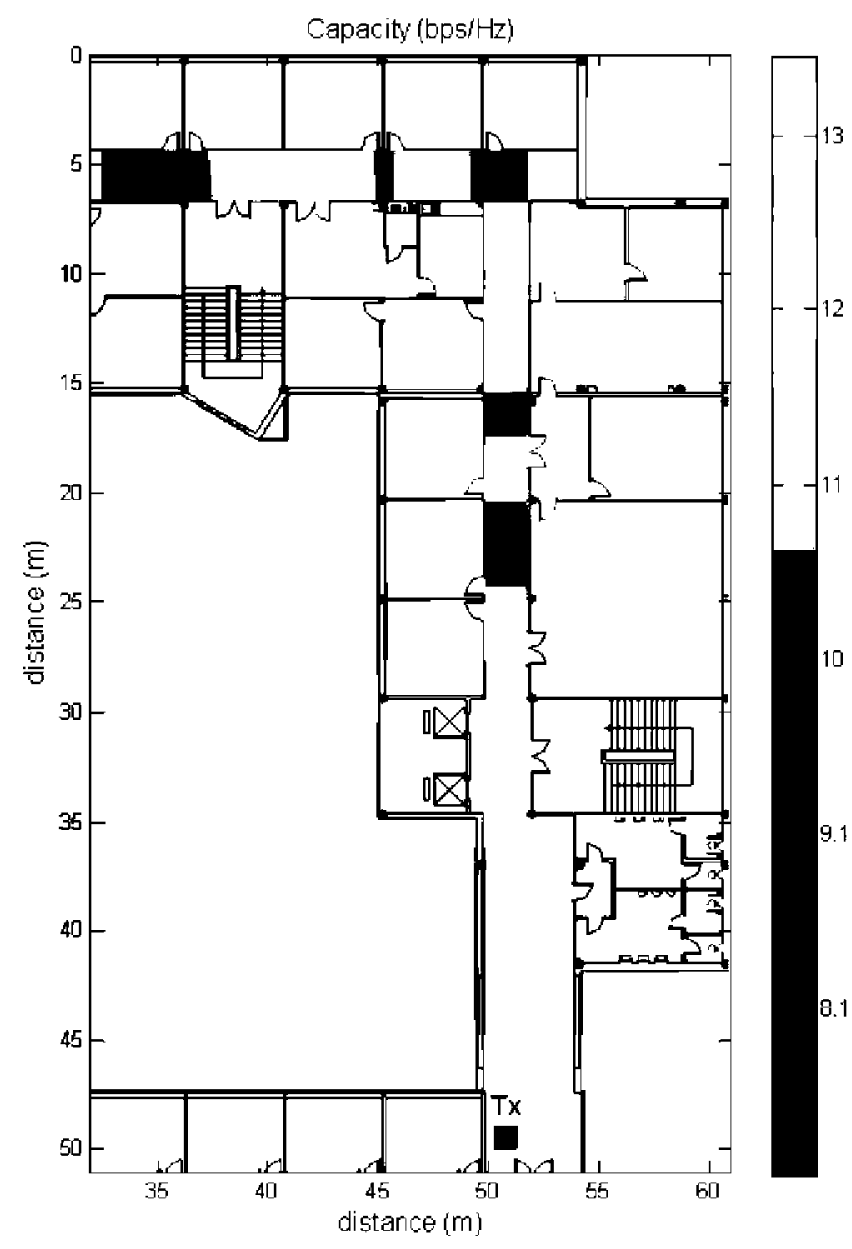

Figure 10 Capacity obtained for $4 \times 2$ MIMO scheme with monopoles in transmission and reception

\section{IMPLEMENTATION}

Once the antennas have been designed and the three MIMO configurations have been studied, the configuration 1 has finally been implemented taking into account the antenna as well as the PDA terminal dimensions. Figure 5 shows the final PDA prototype with the MIMO antennas. Moreover, Figures 6 and 7 represent the comparison of simulated and measured $S$ parameters for the case of antenna 1 (the left one of the Fig. 5). The measurements and simulations of $S$ parameters present quite similar results. For the reflection coefficient, the measurement presents a better coefficient at $2.45 \mathrm{GHz}$, however, the resonant frequency at $5.3 \mathrm{GHz}$ is shifted to $5.4 \mathrm{GHz}$, and both are within the limits designed by the WLAN band. Moreover, the reflection coefficient with a bandwidth at $S_{11}$ $=-6 \mathrm{~dB}$ (typical for compact antennas) of 270, 330, and 328 $\mathrm{MHz}$ for each frequency band $(1800,2450$, and $5300 \mathrm{MHz}$, respectively). On the other hand, Figures 8 and 9 depict the radiation pattern measured of the antenna 1 and 2 , respectively. They show the azimuth plane for different angles of elevation $\left(\theta=0^{\circ}, 45^{\circ}\right.$, and $90^{\circ}$ ). The $x$ axis is vertical in Figure 5 and goes from top to down. The $y$ axis is horizontal in Figure 5 and it is represented from left to right. The difference in radiation of these antennas lies in the ground plane conditions since the antennas are symmetrically placed in the PDA.

\section{MIMO MEASUREMENTS}

In order to evaluate the compact array implemented, MIMO channel measurements have been carried out. The PDA with the tri- band antenna array is connected to the receiver of UMAT (UPM Multi-Antenna Testbed) [7] and an array of four monopoles with a spacing of half wavelength is placed in the transmitter side. The measurements were realized in an indoor scenario comparing the PIFAs to the monopole antennas in a corridor scenario with a $4 \times$ 2 MIMO configuration. The transmitter was situated at the end of a corridor, pointing at the other end while the receiver was moved along the corridor. The $\mathbf{H}$ channel matrix was obtained and normalized to instantaneous Frobenius norm and the capacity for each case was calculated using the expression in (3) with a SNR of $20 \mathrm{~dB}$.

Figures 10 and 11 show the capacity obtained for the case of monopoles and antenna under test (PIFAs), respectively. The implemented PIFAs offer higher capacity in average, even in a situation when the receiver is in front of the transmitter and it is moving from the end to near it. In the case of having a signal with $20 \mathrm{MHz}$ of bandwidth and taking the $10 \%$ of the $\mathrm{CDF}$, the monopoles configuration gives a capacity of $180 \mathrm{Mbps}$ and the PIFA one, 187 Mbps.

\section{CONCLUSION}

In this article, a tri-band Planar Inverted-F Antenna has been presented for WLAN and GSM MIMO applications. The PIFA has been designed for a hand-held user terminal such as PDA. The reflection and coupling coefficients simulated and measured offers high similarity.

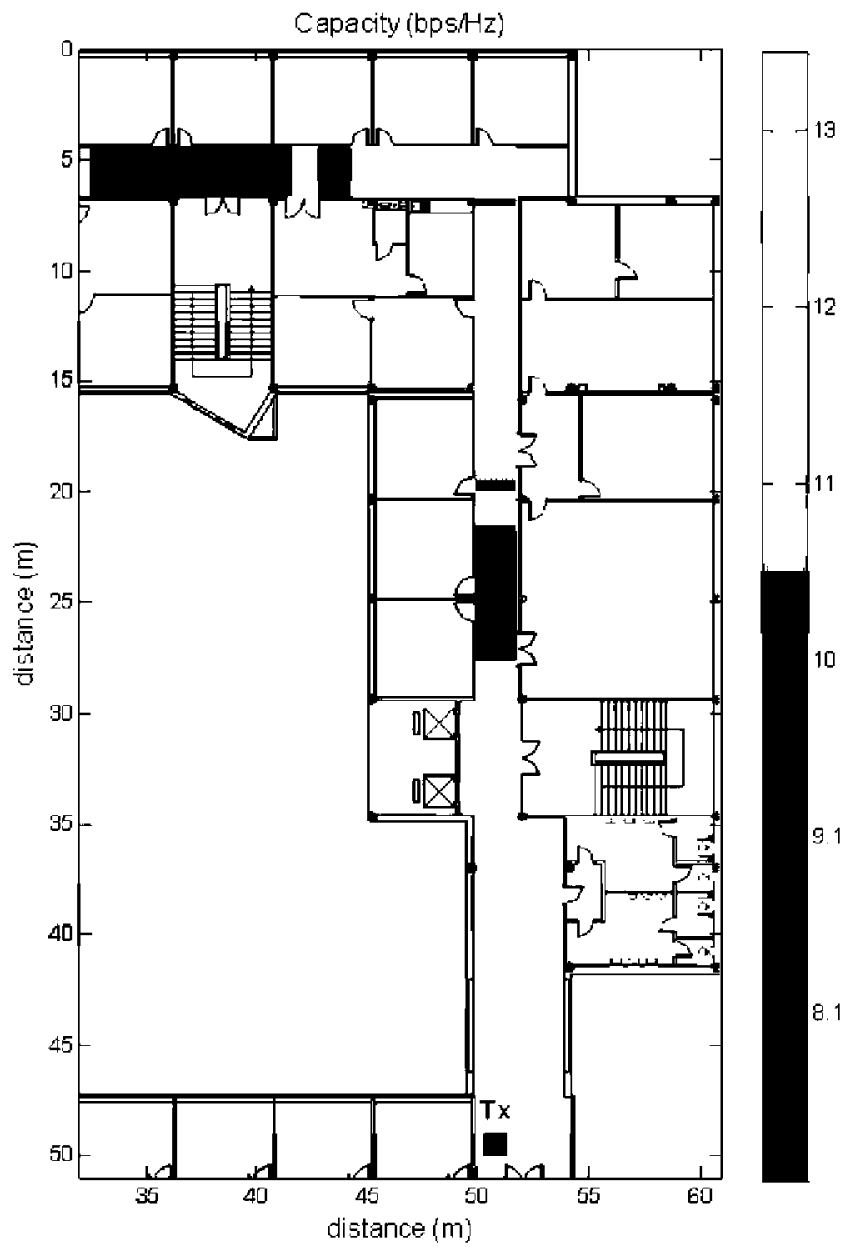

Figure 11 Capacity obtained for $4 \times 2$ MIMO scheme with monopoles in transmission and PIFAs in reception 
On the other hand, several MIMO configurations have been considered to compare performances in terms of channel capacity including electromagnetic parameters of the antenna, such as radiation patterns and mutual coupling. The Spatial Channel Model from 3 GPP has been used for the simulations. Higher capacity has been obtained in the configuration where the two antennas has been placed in parallel with a spacing of 0.4 wavelengths within a PDA, mainly due to the lower mutual coupling and thus to uncorrelation between MIMO subchannels. Moreover, the radiation pattern for both antennas has been measured and MIMO channel measurement have been carried our in an indoor environment, obtaining in average higher capacity in the case of the designed PIFAs.

\section{ACKNOWLEDGMENT}

The authors wish to thank S.R.F. Moyano, from Dragados Industrial, especially to Mr. Alberto Martínez Ollero, for the support of this research work conducted as part of the PIDEA SMART project and partially funded by PROFIT FIT-330210-2005-107. The electromagnetic simulations presented in this article have been realized using CST Microwave Studio version 5.0 under a cooperation agreement between Computer Simulation Technology (CST) and Universidad Politécnica de Madrid.

\section{REFERENCES}

G. Foschini and M. Gans, On limits of wireless communications in a fading environment when using multiple antennas, Wireless Personal Commun 6 (1998), 311-335.

A.J. Paulraj, D.A. Gore, R.U. Nabar, and H. Bölcskei, An overview of MIMO communications-A key to gigabit wireless, Proceedings of the IEEE 92 (2004), 198-217.

K.-L. Wong, Planar antennas for wireless communications, Wiley, New Jersey, 2003.

P. Salonen, M. Keskilammi, and M. Kivikoski, New slot configurations for dualband planar inverted-F antenna, Microwave Opt Technol Lett 28 (2001), 293-298.

3GPP, Spatial channel model for multiple input multiple output (MIMO) simulations, 3GPP TR 25.996 V6.1.0, 2003 [online]. Available: http://www.3gpp.org/ftp/Specs/html-info/25996.htm.

G.J. Salo, J. Salmi, P. Kyösti, M. Milojevic, D. Laselva, and C. Schneider, MATLAB implementation of the 3GPP spatial channel model, 3GPP TR 25.996, 2005 [online]. Available: http://www.tkk.fi/ Units/Radio/scm/.

C. Gómez-Calero, L. Garc, ía-García, R. Martínez, and L. de Haro, Comparison of antenna configurations in different scenarios using a wideband MIMO testbed, IEEE International Symposium 2006 on Antennas and Propagation, Albuquerque, NM, 9-14 July 2006, pp. 301-304. 